\title{
ACKNOWLEDGMENT
}

\section{Referees for Volume 15 (Issues 1-4), 2020}

\author{
(c) Konrad Lorenz Institute for Evolution and Cognition Research 2020
}

We wish to thank the following scholars who have generously shared their time and expertise to serve as reviewers for Biological Theory over the past year. We are truly grateful for their help, as well as for the assistance from reviewers who chose to remain anonymous.

Riadh Abed

Ramsey Affifi

Jan Baedke

Ann-Sophie Barwich

Ramray Bhat

Marion Blute

Daniel Brooks

Zachary Burton

Andrew Buskell

Richmond Campbell

Gustavo Caponi

Linnda Caporael

Charles Carter

Bernard Chapais

Mathieu Charbonneau

Sam Clarke

Gregory Cooper

Sophie de Beaune

Andreas De Block

Nei De Freitas Nunes-Neto

Eric Desjardins

Antoine Dussault

Charbel El-Hani

Niles Eldredge

Paul Ewald

Alan Fiske

Q. Fuller

Bruce Glymour

James Griesemer
Joel Hagen

Manuel Heras-Escribano

Philippe Huneman

Sverker Johansson

Richard Joyce

Jonathan Kaplan

Anton Killin

Eugene Koonin

Aaron Kozbelt

Ádám Kun

Robert Layton

Tim Lewens

Robert Lickliter

Sam Lin

Katherine Liu

Francesca Merlin

Irina Mikhalevich

Roberta Millstein

Alessandro Minelli

John Mitani

Donald Morrison

Gerd Müller

Parashkev Nachev

Karl Niklas

Michael O'Brien

D. Kimbrough Oller

Jun Otsuka

Karenleigh Overmann

Emily Parke
Trevor Pearce

Makmiller Pedroso

Jeffrey Peterson

Charles Pigden

Ronald Planer

Arnaud Pocheville

Paolo Rognini

Nils Roll-Hansen

Joan Roughgarden

Sophia Rousseau-Mermans

Isabella Sarto-Jackson

Roberto Serra

Joan Silk

Subrena Smith

Vassiliki Betty Smocovitis

Yoav Soen

Kim Sterelny

Sonia Sultan

Kristen Syme

Stéphane Tirard

Tobias Uller

Denis Walsh

Rasmus Winther

Publisher's Note Springer Nature remains neutral with regard to jurisdictional claims in published maps and institutional affiliations. 